\title{
Morpho-Pathological Review on the Healing of Synthetic Vascular Grafts
}

\author{
Russu Eliza1 ${ }^{\star}$, Mureșan Adrian Vasile, Cordoș Bogdan Andrei², Copotoiu Constantin¹, Cotoi Ovidiu \\ Simion ${ }^{3}$
}

1 Department of Surgery, University of Medicine and Pharmacy of Tirgu Mures, Romania

2 Experimental Research Station, University of Medicine and Pharmacy of Tîrgu Mureș, Romania

${ }_{3}^{3}$ Pathophysiology Department, University of Medicine and Pharmacy of Tîrgu Mureș, Romania

Objective: Tissue integration of vascular grafts partially depends on the host response to injury, which immediately begins after implantation and restoration of the circulation. In an infected environment, the inflammation changes the incorporation patterns. The aim of the study was to observe the tissue incorporation process, in a normal and an infected environment. Methods: We have created an experimental model by performing subfascial implantation of four types of vascular grafts, in rats (woven Dacron $®$, knitted Dacron ${ }^{\circledR}$, silver coated Dacron $®$ and expanded Polytetrafloroethylene - ePTFE) and by infecting some of them with three different bacterial strains. We have retrieved the noninfected grafts at two and four weeks after implantation, whilst the infected ones at one, two and three weeks. Results: Detailed microscopic appearences were analysed. The control and infected groups were compared. Statistical significance was calculated for various corelations. Conclusions: The morphopathological findings showed that the ePTFE graft's structure was best preserved. Statistical significance existed between the bacterial strain and the degree of inflammation. The silver coated Dacron $₫$ was not shown to be superior to the knitted Dacron ${ }^{\circledR}$. The poorest incorporation was the one of the woven Dacron ${ }^{\circledR}$.

Keywords: grafts, infection, microscopy, inflammation

Received: 14 August 2015 / Accepted: 11 September 2015

\section{Introduction}

Tissue integration of the vascular graft partially resides on the host response to injury, which immediately begins after implantation and restoration of the circulation. The bloodgraft interface [1] creates a complex reaction cascade, starting with the adsorption of plasma proteins and platelet deposition and followed by infiltration of neutrophils and monocytes and eventually proliferation of endothelial and smooth muscle cells.

Even the most non-reactive, biologically inert materials are still recognized by the human body as "non-self" or "foreign".

Current vascular surgery methods use grafts such as woven and knitted Dacron ${ }^{\ominus}$, silver coated Dacron ${ }^{\circledR}$ and expanded Polytetrafluoroethylene (ePTFE), known as Gore$\mathrm{Tex}^{\oplus}$, and improved varieties as: colagen sealed, heparin or antimicrobial substances bonded or carbon-impregnation.

The ePTFE grafts are considered to be more biologically inert than the polyethylene terephthalate $\left(\mathrm{Dacron}^{\circ}\right)$ ones.

Once the blood flow through the graft, after declamping, has been re-established, the protein adsorption starts, as well. Fibrinogen, albumin and immunoglobin $\mathrm{G}(\mathrm{IgG})$ are being adsorbed instantly, and then a redistribution of proteins is taking place, ordered by each protein's electrical and biochemical affinity for the surface of the graft [2].

Other plasma proteins, such as complement components, may be activated by the "non-self" substance of the

${ }^{*}$ Correspondence to: Eliza Russu

E-mail: eliza.russu@umftgm.ro synthetic graft. The complement component C5a generation (which is a powerfull monocyte chemoattractant) has experimentally been proven to be greater after $\mathrm{Dacron}^{\circledR} \mathrm{im}$ plantation, compared with ePTFE [3].

Platelets early deposition is achieved via the adsorbed proteins on the surface of the graft. After adherence, platelets will degranulate, releasing substances such as adenosine diphosphate, serotonin, epinephrine, tromboxane A2, which will themselves activate other platelets and will increase the generation of thrombin.

The activated platelets release growth factors such as platele-derived growthfactor (PDGF) and transforming growth factor $\beta$ (TGF- $\beta$ ), which modulate the migration and proliferation of endothelial cells (EC) and of smooth muscle cells (SMC), as well as the synthesis and degradation of the extracellular matrix. Platelets deposition and activation continues for more than one year after graft implantation [4].

Acute inflammatory response is mediated through C5a and B4 leukotriene, which attract neutrophils onto the surface of the graft. The neutrophils are attracted to the fibrin coagulum formed on the inner or outer surface of the graft. But the recruitment of these phagocytic cells has no impact on the resistance to infection. On the contrary, these adherent neutrophils show little phagocytic capabilities, not being able to properly kill germs. In the sites which have been activated by interleukin 1 (IL-1) and tumor necrosis factor (TNF) the monocytes are also atracted, being then differentiated into macrophages and being the main participants in chronic inflammmatory response. The activated 
macrophages form giant multinucleated cells, the foreign body cells, which can be found lining the graft, a process which is called "frustrated phagocytosis" [5].

Monocytes and macrophages incubated with Dacron ${ }^{\circ}$ have been shown to produce a higher amount of IL-1 $\beta$, TNF and IL-6 than the ones incubated with ePTFE [6].

After graft implantation the tissue ingrowth starts from the anastomosis. In animal models [7] the complete endothelialization of the synthetic grafst was observed, whereas in humans there are limits of the EC ingrowth, which stops in $1-2 \mathrm{~cm}$ of the anastomosis, being found again in the mid-parts of the length of the grafts as endothelial islands, which suggests alternative sources of EC, provided by interstitial tissue ingrowth, for example, in Dacron $^{\bullet}$ grafts (which exhibit a higher porosity). Other sources may also be bone-marrow derived endothelial progenitor cells [8], which are released in the blood stream as a response to stimuli such as ischemia [9] or vascular trauma.

Once activated [10], endothelial cells release bioactive substances which furthermore promote thrombogenesis and the SMC ingrowth. The subintimal SMC proliferation takes place in areas located right near the anastomosis, areas defined by complex biomechanical characteristics and marked by a chronic inflammatory injury.

SMC proliferation begins 2 days after the graft's implantation, reaches a peak by 2 weeks and then fades away, although without complete dissapearence.

When synthetic graft infection appears, the prosthesis and the adherent bacteria, generally found in a biofilm, act as one, forming an inflammatory stimulus for the immune system, for the pro-inflammatory cytokines [11]. This results in an inflammatory process which tries to localize the infection, but induces tissue-damaging effects, such as the recruitment of polymorphonuclear granulocytes and the production of other cytokines. Synthetic grafts, unlike autologous ones, do not develop vascular connections with the perigrafts tissues, which prevents host defense from reaching maximum potency.

At the beggining, the inflammation surrounding the infected graft only produces a failure of graft healing and forms a perigraft cavity or an abscess. As the infection spreads, other structures (muscles, bowel, skin) may be affected. This pathobiology [12] is clinically manifested (often by a high grade Szilagyi wound infection), but if the involved bacteria are of the"low-virulence" type, then a late infection [13] (over 4 months after implantation) may be suspected by the signs of failure in graft incorporation, in this case the perigraft fluid and tissues not containing germs, only large numbers of white blood cells.

\section{Method}

We have used 120 Wistar rats, aged between 4-5 months, weighing aproximately 500 grams, originated from the Cantacuzino Institute Experimental Station, divided into two groups: a control group and an infected group (later sub-divided into other groups).
The rats were kept in special polycarbonate cages, according to the European Union Guidelines, were quarantined for one month towards acclimatization and were fed with granulated fodder ad libitum. The study was approved by the Scientific Research Ethics Commitee of the University of Medicine and Pharmacy Targu Mures, decision number 73/20.07.2015.

We have anesthetized the rats by intraperitoneally injecting them with Ketamine $75 \mathrm{mg} / \mathrm{kg}$ and Xylazine 10mg/ $\mathrm{kg}$.

We have surgically draped a skin portion of 5 by 5 centimeters, in the interscapular region. Through a longitudinal skin incision of approximately 3 centimeters on the median line and the dissection of the tissues, we have implanted a 2 by 2 centimeters prepared portion of a synthetic graft, either woven, knitted, silver coated Dacron ${ }^{\circledR}$ or ePTFE, sutured subfascial.

In 80 of the 120 rats we have infected the grafts by direct inoculation of 0.3 mililiters of one of the standardized strains: methicillin-sensitive Staphylococcus aureus (MSSA) - American Type Culture Collection (ATTC) 29213 (subspecies aureus Rosenbach, strain designation: Wichita), methicillin-resistant Staphylococcus aureus (MRSA) ATTC 43300 (subspecies aureus Rosenbach, strain designation: F-182) and Escherichia coli (E.coli) ATCC 25922 (Migula 1895, Castellani and Chalmers 1919, strain designation: FDA strain Seattle 1946) on the surface of the graft, before closing the wound.

The diagram of the sub-divisions of the control and infected groups of rats is presented in figure 1.

These data were partially used in a second article, submitted for publication, pertaining to the same research project.

Half of the non-infected, control group of rats' grafts was retrieved at 2 weeks after implantation, whilst the other half after one month.

The first sub-group of the MSSA infected rats was removed from the study, the bacterial inoculum being too weak to provide an infection.

The second sub-group of MSSA infected rats' grafts was retrieved at 2 weeks.

Then other 3 sub-groups were infected with MSSA and MRSA and the grafts were retrieved at 7 days, 14 days and 21 days. Also 3 sub-groups were infected with E.coli and the grafts have been retrieved following the same rule of thumb: 7, 14 and 21 days after the implantation.

In conclusion, we had 10 sub-groups, each containing 8 rats, 4 of these sub-groups being infected with MSSA, 3 infected with MRSA and 3 infected with E.coli. All grafts have been retrieved at one, two and three weeks of evolution, except for 2 sub-groups infected with MSSA having the grafts retrieved at two weeks both. The explanation for this excedent is the change in the design of the sub-groups in the attempt of a better glimpse of each infection's pattern of evolution if followed closely, within seven days' distance in retrieval. 


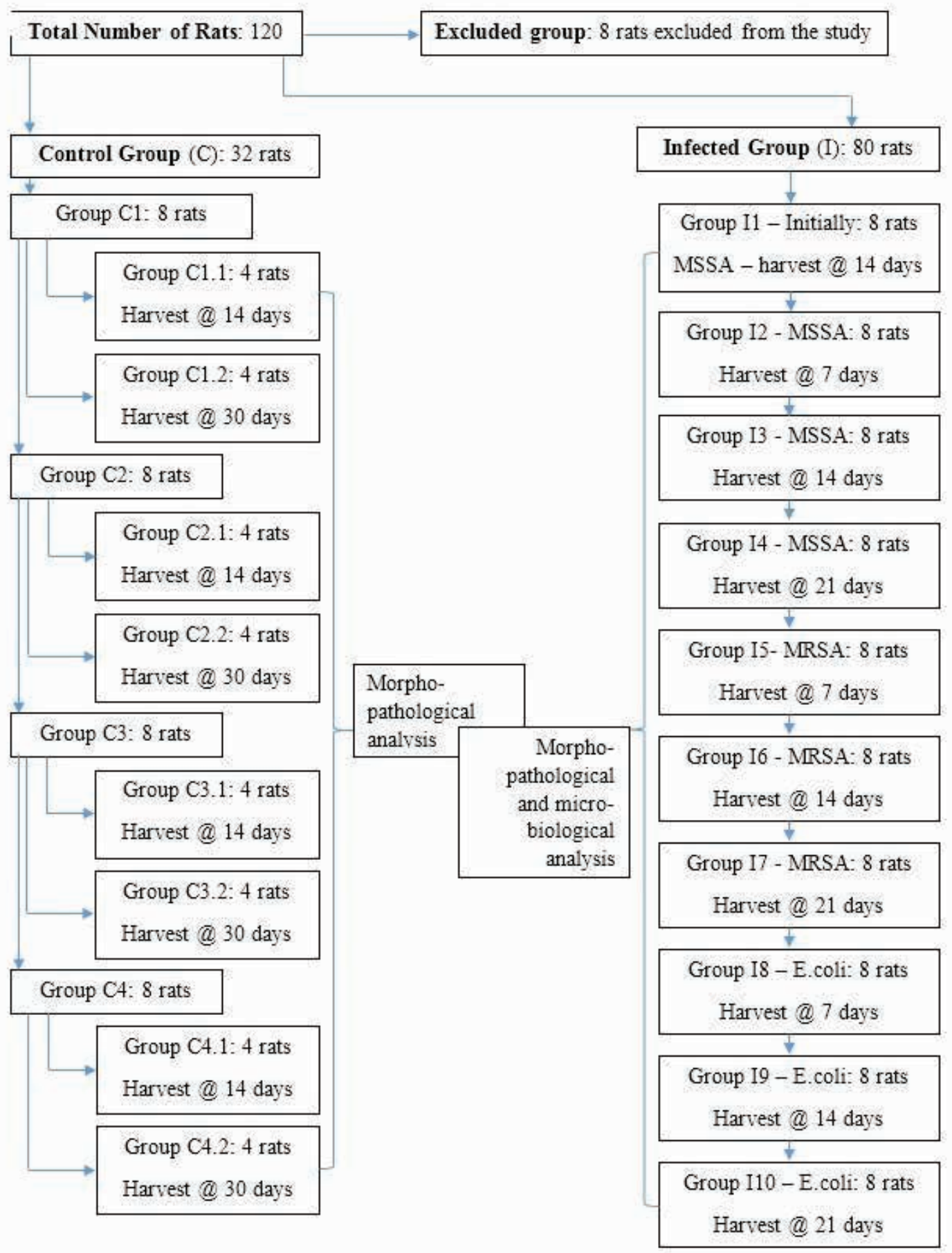

Fig. 1. Diagram of rat groups divisions: Diagram showing the distribution of the rats used in the study, based on type of implant, infectious agent and implant retrieval.

The retrieval of the grafts was made through euthanasia, done as follows: first we have anesthetized the rats by injecting them with Ketamine $75 \mathrm{mg} / \mathrm{kg}$ and Xylazine $10 \mathrm{mg} /$ $\mathrm{kg}$ and then we have administrated intracardiac 0,1 mililiters of T61 (embutramide $200 \mathrm{mg}$, mebezonium iodide $50 \mathrm{mg}$, clorhidric tetracaine $5 \mathrm{mg}$ ), which resulted in cardiac arrest within 10-20 seconds.

Tissue and graft fragments were processed using standardized histologic techniques: $4 \%$ formol fixation for 24 hours, paraffin embedding, microtome sectioning and hematoxylin and eosin staining. The microscope examining and data acquisition was done using a Nikon Eclipse E-600 microscope.
The extracted grafts were morphopathologically analyzed, first the non-infected ones which were extracted at 2 and 4 weeks after implantation, then the infected ones, which were extracted at one, two and three weeks after implantation. The structures were compared, and also the way the grafts reacted to infection, and the time-depending changes in cellular populations surrounding the grafts were noted.

A semi-quantitative evaluation method was established to evaluate the degree of inflammation, with grades ranging from 1 to 3 , where 1 defined minor inflammation, 2 moderate inflammation and 3 intense inflammation. 
Statistical analysis of the results was aimed to look for the following corelations and their statistical significance: graft invasion according to each bacterial type, graft structure integrity according to bacteria, Szilagyi degree of the surgical site infection according to each bacteria and to the day of graft retrieval. The data were analyzed using the EpiInfo7 statistical program. Chi-aquare test was used, with significance level 0.05 .

\section{Results}

There was no statistical significance between graft invasion and bacterial type. However, there was statistical significance between the preservation of the structure of the graft and the bacterial type. MSSA infection was not able to produce invasion or disruption of the structure of the synthetic grafts, whereas the more virulent MRSA and E.coli infections invaded the grafts and partially disrupted the structute of the Dacron ${ }^{\circ}$ grafts. Though the ePTFE grafts were invaded by inflammatory cells, which were able to penetrate through the structure when severe infection took place, their structure was constantly preserved, as opposed to the Dacron ${ }^{\ominus}$ grafts. The preservation of Gore-Tex ${ }^{\oplus}$ graft structure was statistically significant, in compared to the Dacron ${ }^{\oplus}$ grafts.
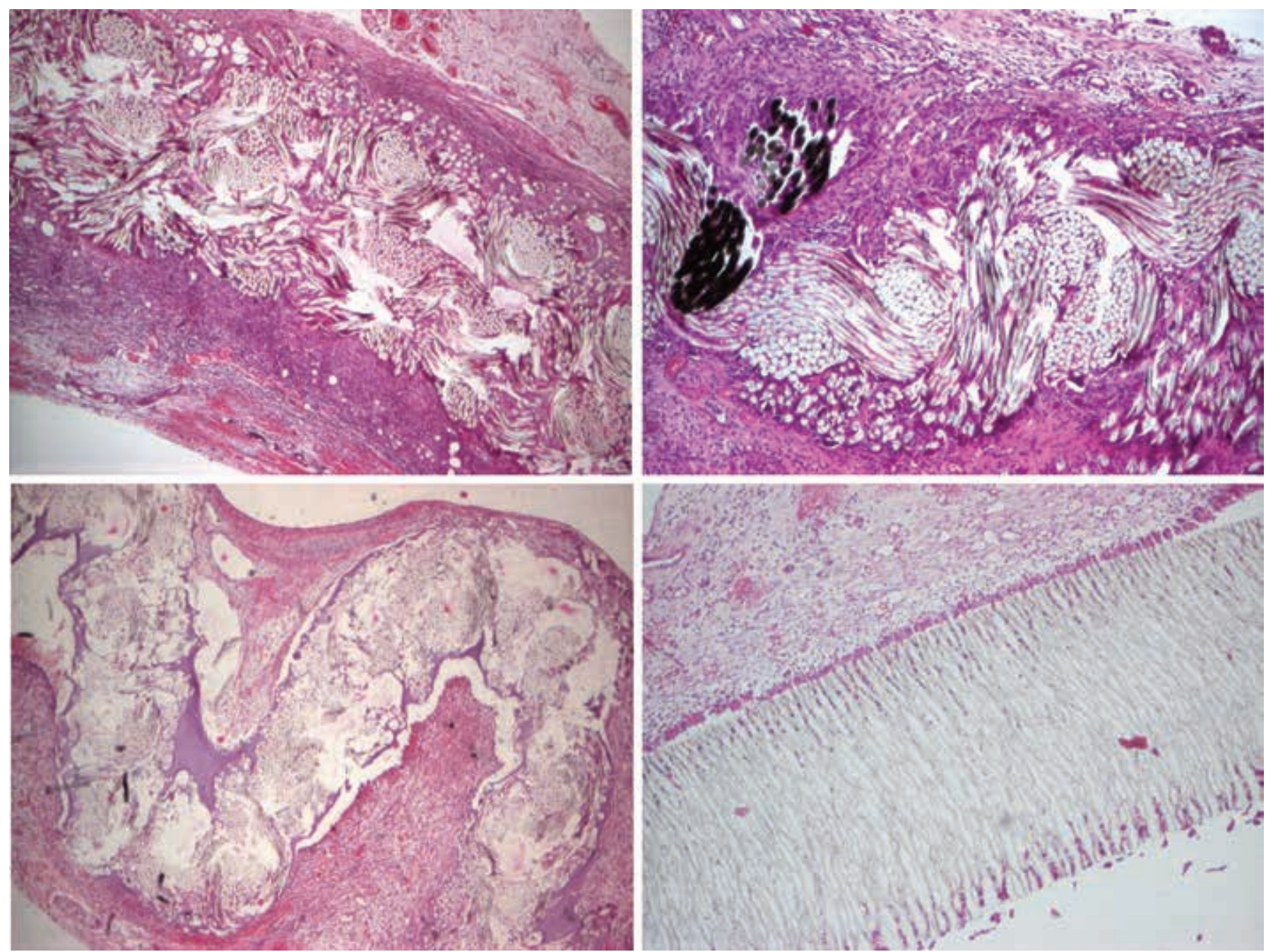

Fig. 2. Control Group Microscopy: Microscopic appearence of the control group. Hematoxylin and eosin staining, $\mathrm{x} 4$ objective lenses. Upper left: silver coated Dacron®; upper right: woven Dacron $\AA$; lower left: knitted Dacron®; lower right: Gore-Tex®.
There was also statistical significance between bacterial type and the Szilagyi degrees of wound infection (grade 1 Szilagyi defining the cellulitis involving the wound, grade 2 Szilagyi-infection involving the subcutaneous tissue and grade 3-infection involving the vascular graft). The most grade 3 Szilagyi infections were produced by MRSA and E.coli. The distribution patterns, according to the harvesting of the graft, were different, as follows: the more aggresive MRSA strain has produced, from the very beggining, a grade 3 Szilagyi wound infection which affected mostly the Dacron ${ }^{\bullet}$ grafts and had no healing trend as days passed. The E.coli strain equally affected all types of grafts, but did not produce, for grafts retrieved at the end of the first week, a high Szilagyi grade. As time passed, in grafts retrieved at the end of second and third week, there was noticeable aggravation in wound evolution, with the appearence of peri-graft reactions and tissue destructions (crossing the limit of the fascia, observed at extraction) qualifing for grade 3 Szilagyi infection. For grafts harvested at 14 and 21 days from implantation, statistical significance was obtained between Szilagyi grade and bacterial type.

Despite intense inflammation (especially in the case of E.coli infections, which produced positive blood cultures 
and one death caused by acute respiratory distress syndrome) and graft invasion by inflammatory cells, as exemplified in the following pages, the overall structure of the ePTFE graft remained unmodified. Statistical significance has been observed between the bacterial strain and the degree of inflammation.

There was no statistical significance found between the degree of inflammation and the day of harvest.

\section{Detailed miscroscopical description of the control group}

For all 3 types of Dacron': woven, knitted and silver coated, the miscroscopic aspect was similar. The synthetic grafts were embedded in a granulation tissue with numerous neoformation blood vessels developed during the first two weeks. As for the grafts retrieved after 4 weeks, these were embedded in a fibrous tissue. The exudative phase of inflammation was still present in grafts retrieved at 2 weeks, whilst for the others, chronic inflamamatory infiltrate took place, abundant in lymphocytes and histiocytes, along with giant body, multinucleate cells, which formed altogheter with the infiltrate and the tissue re- action foreign body granulomas surrounding the grafts. Woven Dacron ${ }^{\circledR}$ grafts suffered the biggest changes from a structural and micro-architectural point of view, having a plied appearance and focally presenting numerous breaking areas. These areas may be partially due to microtome technique used in processing. Knitted and silver coated Dacron $^{\oplus}$ kept their micro-architecture unbroken, without significant changes during processing. ePTFE grafts had the best preserved structure, without any breaking points. Histologically, the synthetic graft was isolated by the body, surrounded by numerous foreign body giant cells lining the surface of the graft. The inflammatory infiltrate was missing and the perigraft tissues were slightly edematous and showed small blood vessels proliferation (Figure 2).

\section{Detailed miscroscopical description of the infected group}

For the Dacron ${ }^{\circledR}$ grafts two types of tissue reactions were observed microscopically: a granulomatous reaction (a foreign body-like reaction) and an infectious reaction due to MSSA, MRSA and E.coli.
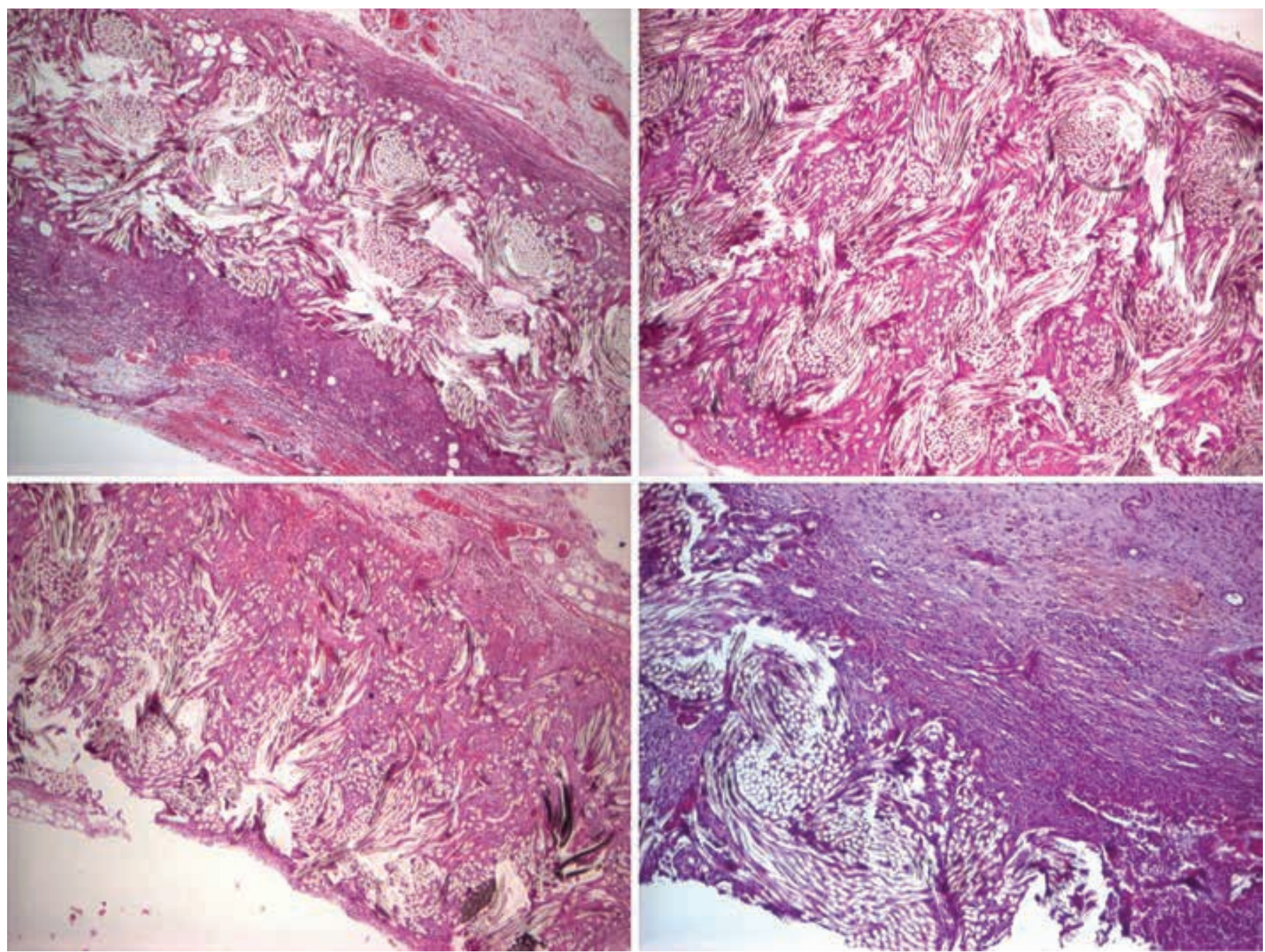

Fig. 3. Comparative Silver Coated Dacron $₫$ Microscopy: Microscopic appearence of the infected silver coated Dacron $®$ graft, compared with the control group appearence. Hematoxylin and eosin staining, $x 4$ objective lenses. Upper left: silver coated Dacron $\circledast$ graft from the control group; Upper right: silver coated Dacron $₫$ MSSA infected; lower left: silver coated Dacron® MRSA infected; lower right: silver coated Dacron ${ }^{\circledR}$ E.coli infected. 
The granulomatous reaction followed the described pattern for the control group, only with a faster appearence of granulomas and a higher extent of graft destruction. The most badly afflicted were the woven Dacron ${ }^{\circ}$ grafts, followed by the knitted Dacron ${ }^{\circledR}$ and then the silver coated ones. The infectious reaction showed itself through the appearence of segmented neutrophils, along with lymphocytes, macrophages and giant multinucleate cells.

The MSSA infection produced a mild polymorphous inflammatory infiltrate, whilst MRSA and E.coli infections produced wide areas of suppuration. This suppurative appearence was found in all the Dacron ${ }^{\bullet}$ grafts (woven, knitted and silver coated). In the Gore-Tex ${ }^{\bullet}$ grafts the MSSA infection produced smaller giant multinucleate cells and some small histiocytes or lymphocytes which could be observed among the free spaces of the graft fibers. In the control group, inflammatory and giant cells stopped at the edge of the synthetic graft. In contrast, in the infected grafts, inflammatory cells infiltrated and penetrated the whole thickness of the graft. The MRSA and E.coli infections additionally produced suppuration: all around the graft inflammatory infiltrate was found, dominated by the massive presence of the segmented neutrophils, as well as edema and surrounding tissue necrosis (Figure 3-6).

\section{Discussions}

The physio-pathological mechanisms involved in tissue integration of synthetic vascular grafts include type II (cytotoxic) hypersensitivity reactions and type III (immune complexes) reactions, according to the Gell and Coombs classification. These mechanisms explain the acute inflammmatory reaction and infection mediation.

Whenever foreign body granulomas formation occurs, type IV late reactions, cell-mediated immunity tributary ones are activated, with the implication of T-lymphocytes and macrophages, which transform themselves into multinucleate giant cells.

The mechanisms of inflammation and infection of implanted grafts are influenced by the host response to injury[14],[15] and by the type of synthetic polymer as well[16], but as Kevin Geary et al demonstrated in an experimental work[17], graft material appears to be less important than bacterial virulence.
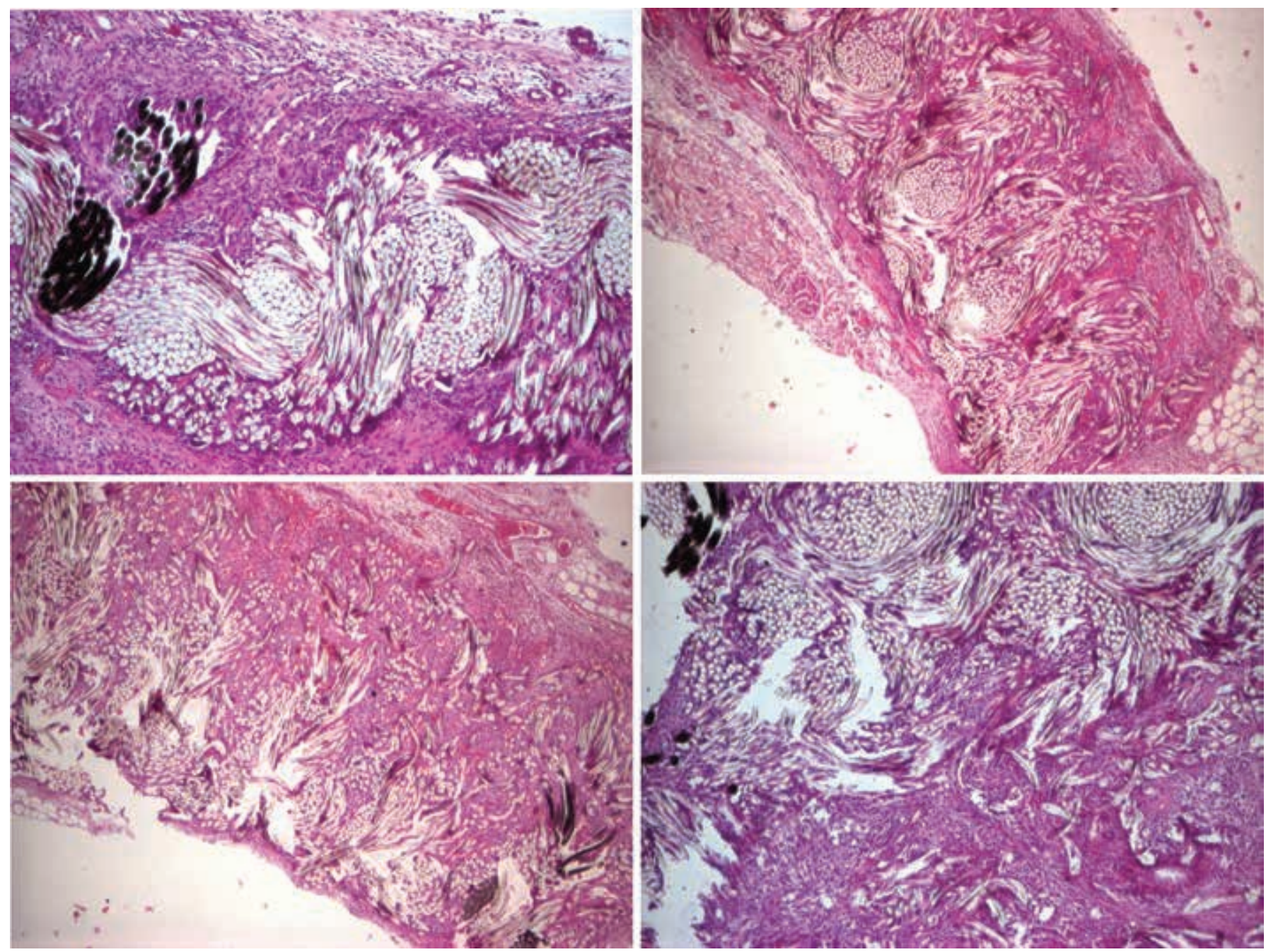

Fig. 4. Comparative Woven Dacron $₫$ Microscopy: Microscopic appearence of the infected woven Dacron $₫$ graft, compared with the control group appearence. Hematoxylin and eosin staining, $x 4$ objective lenses. Upper left: woven Dacron $®$ graft from the control group; upper right: woven Dacron ${ }$ MSSA infected; lower left: woven Dacron $®$ MRSA infected; lower right: woven Dacron $®$ E.coli infected. 

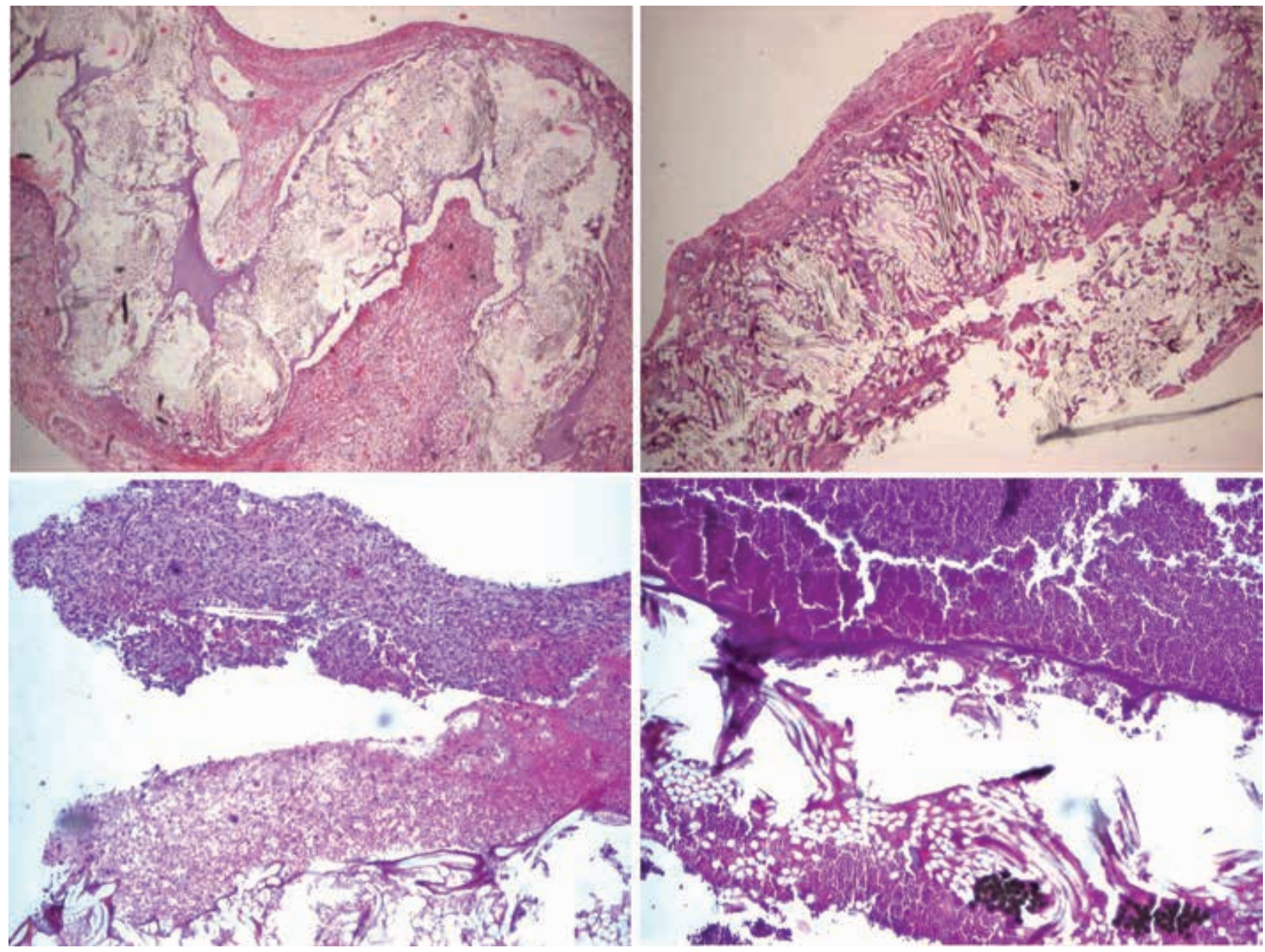

Fig. 5. Comparative Knitted Dacron $®$ Microscopy: Microscopic appearence of the infected knitted Dacron® graft, compared with the control group appearence. Hematoxylin and eosin staining, $x 4$ objective lenses. Upper left: knitted Dacron $\circledast$ graft from the control group; upper right: knitted Dacron ${ }$ MSSA infected; lower left: knitted Dacron $₫$ MRSA infected; lower right: knitted Dacron $®$ E.coli infected.

\section{Conclusions}

Statistical significance has been observed between the bacterial strain and the degree of inflammation. The "frustrated phagocytosis" process was highlighted in this study, especially linked to the ePTFE graft. Despite intense inflammation and graft invasion by inflammatory cells, the overall structure of the ePTFE graft remained unmodified. Healing and tissue incorporation differences between the woven, knitted and silver coated Dacron ${ }^{\oplus}$ are not significant, although the woven graft has the poorest behavior and produces the most extensive granulomatous reactions.

\section{Acknowledgements}

Research reported in this publication was funded by the European Social Fund, within the framework of the Sectoral Operational Programme Human Resources Development 2007-2013, under award number: POSDRU/19/1.5/S/136893.

Any opinions, findings, and conclusions or recommendations expressed in this material are those of the Authors and do not necessarily reflect the views of the institutions mentioned throughout the manuscript.
We thank our colleagues from the University of Medicine and Pharmacy of Târgu Mureș who provided insight and expertise that greatly assisted the research, although they may not agree with all of the interpretations/conclusions of this paper.

We thank Edit Székely, PhD, MD, Lecturer, Microbiology Department and Anca Delia Mare, PhD, MD, Lecturer, Microbiology Department, for assistance with providing the bacterial strains used in the research.

We would also like to show our gratitude to Marius Petrișor, PhD, MD, Assistant Lecturer, Medical Informatics and Biostatistics Department, for the statistical interpretations and comments that greatly improved the manuscript.

The Author would also like to convey thanks to the University for providing the laboratory and research facilities.

\section{Conflict of interest}

There are no conflicts of interest to declare regarding any of the following: employment, consultancies, stock ownership, equity interests, and patent-licensing arrangements. 


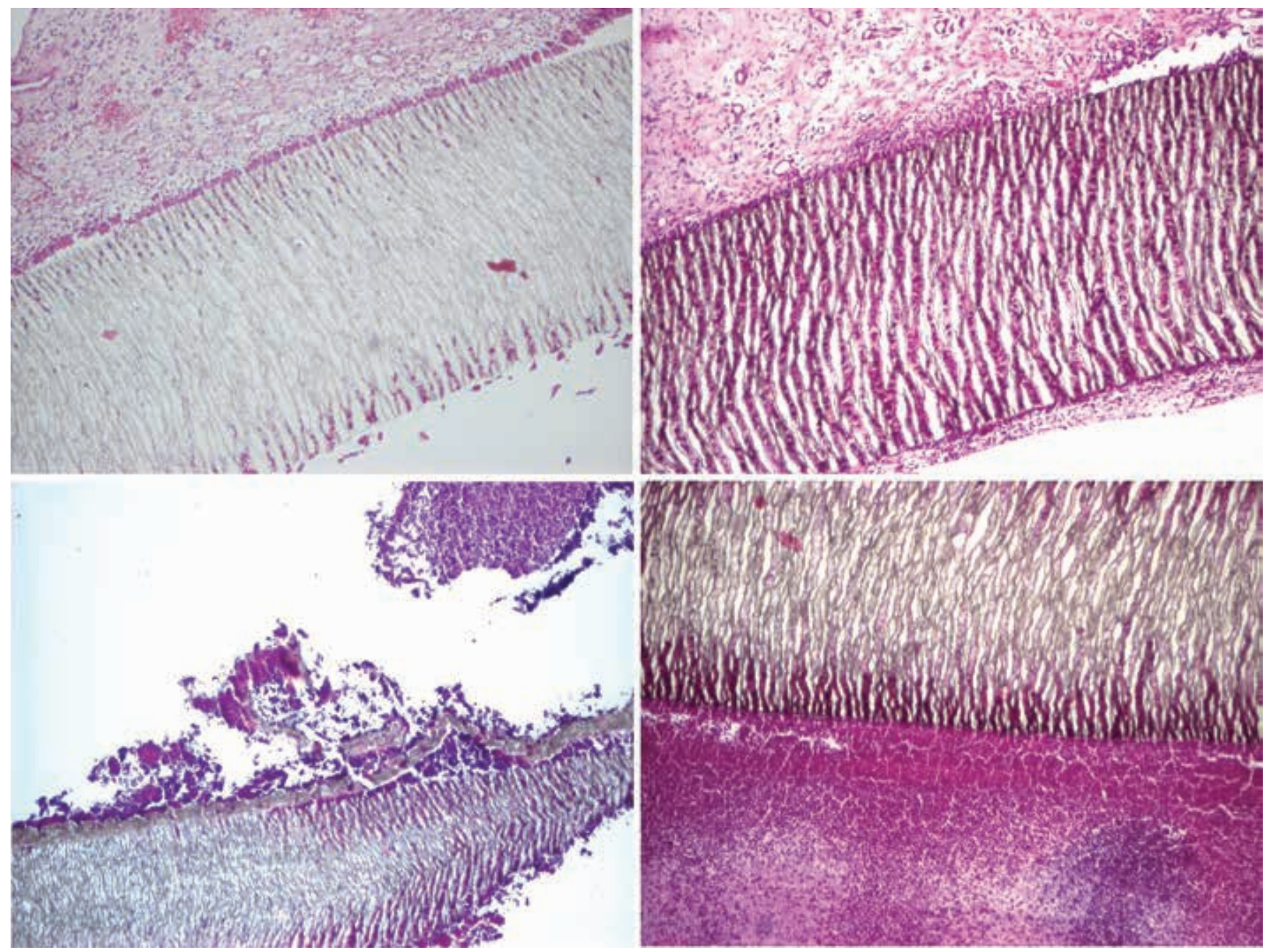

Fig. 6. Comparative Gore-Tex® Microscopy: Microscopic appearence of the infected Gore-Tex® graft, compared with the control group appearence. Hematoxylin and eosin staining, $x 4$ objective lenses. Upper left: Gore-Tex® from the control group; upper right: Gore-Tex® MSSA infected; lower left: Gore-Tex® MRSA infected; lower right: Gore-Tex® E.coli infected.

\section{References}

1. Donovan TJ, Zimmerman B. The effect of artificial surface on blood coagulability, with special reference to polyethylene. Blood. 1949;4:1310.

2. Vroman $L$. Methods of investigating protein interaction on artificial and natural surfaces. Ann N Y Acad Sci. 1978;516:300.

3. Shepard AD, Gelfand JA, Callow AD, O'Donnell TF Jr. Complement activation by synthetic vascular prostheses. J Vasc Surg. 1984;1:829.

4. McCollum CN, Kester RC, Rajah SM, et al. Arterial graft maturation, the duration of thrombotic activity in Dacron aortobifemoral grafts measured by platelet and fibrinogen kinetics. Br J Surg. 1981;68:61.

5. Kzhyshkowska J, Gudima A, Riabov V, et al. Macrophage responses to implants: Prospects for personalized medicine. J Leukoc Biol. 2015; Published online before print July 13, 2015 doi:10.1189/jlb.5VMR0415166R. http://www.jleukbio.org/content/early/2015/07/13/jlb.5VMR0415166R.long

6. Swartbol P, Truedsson L, Parsson H, Norgren L. Tumor necrosis factor- $\alpha$ and interleukin-6 release from white blood cells induced by different graft materials In Vitro are affected by pentoxifylline and iloprost. J Biomed Master Res. 1997;36:400.

7. Raiter A, Bechor Z, Kleiman M, et al. Angiogenic peptides improve blood flow and promote capillary growth in a diabetic and ischaemic mouse model. Eur J Vasc Endovasc Surg. 2010;40(3):381-388.

8. Alobaid N, Salacinski HJ, Sales KM, et al. Nanocomposites containing bioactive peptides promote endothelialisation by circulating progenitor cells: An In Vitro evaluation. Eur J Vasc Endovasc Surg. 2006;32(1):76-83.
9. Cassar K, Bachoo P, Ford I, Greaves M, Brittenden J. Markers of coagulaton activation, endothelial stimulation and inflammation in patients with peripheral arterial disease. Eur J Vasc Endovasc Surg. 2005;29(2):171-176.

10. Armitage JD, Lindsey NJ, Homer-Vanniasinkam S. The role of endothelial cell reactive antibodies in peripheral arterial disease. Eur J Vasc Endovasc Surg. 2006;31(2):170-175.

11. Daryapeyma A, Pedersen G, Laxdal E, et al. Neutrophil CD64 as a marker for postoperative infection: A pilot study. Eur J Vasc Endovasc Surg. 2009;38(1):100-103.

12. Lorenz U, Schäfer T, Ohlsen K, et al. In Vivo detection of Staphylococcus aureus I $\mathrm{n}$ biofilm on vascular prostheses using non-invasive biophotonic imaging. Eur J Vasc Endovasc Surg. 2011;41(1):68-75.

13. Calligaro KD, Veith FJ, Schwartz ML, Dougherty MJ, DeLaurentis DA. Differences in early versus late extracavitary arterial graft infections. J Vasc Surg. 1995;22(6):680-688.

14. Anderson JM. Chapter 4 Mechanisms of inflammation and infection with implanted devices. Cardiovasc Pathol. 1993;2(3):33-41.

15. Zimmerli W, Sendi P. Pathogenesis of implant-associated infection: the role of the host. Sem Immunol. 2011;33(3):295-306.

16. Anderson JM, Rodriguez A, Chang DT. Innate and adaptive immune responses in tissue engineering. Sem Immunol. 2008;20(2):86-100.

17. Geary KJ, Tomkiewicz ZM, Harrison HN, et al. Differential effects of a gram-negative and a gram-positive infection on autogenous and prosthetic grafts. J Vasc Surg. 1990;11(2):339-347. 